\title{
DEFORMATION OF A GENERICALLY FINITE MAP TO A HYPERSURFACE EMBEDDING
}

\author{
FABRIZIO CATANESE AND YONGNAM LEE
}

\begin{abstract}
We give a structure theorem for projective manifolds $W_{0}$ with the property of admitting a 1-parameter deformation where $W_{t}$ is a hypersurface in a projective smooth manifold $Z_{t}$.

Their structure is the one of special iterated univariate coverings which we call of normal type, which essentially means that the line bundles where the univariate coverings live are tensor powers of the normal bundle to the image $X$ of $W_{0}$.

We give applications to the case where $Z_{t}$ is projective space, respectively an Abelian variety.
\end{abstract}

\section{Dedicated to Edoardo Sernesi on the occasion of his 70th birthday}

\section{INTRODUCTION}

Many years ago Sernesi [7] showed that small deformations of complete intersections in projective space, of dimension $n \geq 2$ (the case of curves, $n=$ 1 is of quite different nature, see e.g. [8]), are again complete intersections, unless the complete intersection defines a K3 surface (i.e., $n=2$ and the canonical bundle is trivial). Hence, in particular, smooth hypersurfaces in projective space $\mathbb{P}^{n+1}$ form an open set in the Kuranishi space, respectively an open set in the moduli space when they are of general type, unless $n=$ 2 and the degree equals 4 . In considering the closure of this set in the moduli space, we have to deal with varieties $W_{0}$ of the same dimension, given together with a generically finite rational map $\phi_{0}: W_{0} \rightarrow \mathbb{P}^{n+1}$.

As shown by Horikawa in [5], already in the easiest nontrivial case $n=2$, $\operatorname{deg}\left(W_{0}\right)=5$ the situation becomes rather complicated. But we show here that things are simpler in the case where $\phi_{0}$ is a morphism.

A similar result to Sernesi's holds for hypersurfaces in an Abelian variety (Kodaira and Spencer's theorem 14.4 in [6]), and we can consider the closure of the locus of hypersurfaces $X$ in Abelian varieties (for $n \geq 2$ the Abelian variety is just the Albanese variety of $X$ ) observing that in this case any limit $W_{0}$ has a generically finite Albanese map $\phi_{0}: W_{0} \rightarrow A_{0}$ (see for instance lemma 149 of [3]).

Date: September 24, 2018.

AMS Classification: 14D15, 14J15, 14J70, 14K12, 32G05, 32G10, 32H02.

Key words: Deformation theory, hypersurfaces, embeddings, iterated univariate coverings, Inoue-type varieties.

The present work took place in the framework of the ERC Advanced grant n. 340258, 'TADMICAMT'. Both authors would also like to acknowledge the support and hospitality of KIAS, Seoul which they visited as Research Scholar, respectively as Affiliate Professor. The second author was also supported by Basic Science Research Program through the NRF of Korea (2016930170). 
Also in this case we can ask the question of characterizing the morphisms $\phi_{0}$ admitting a deformation which is a hypersurface embedding in some Abelian variety, deformation of the original one.

The main motivation for posing this question also in higher generality comes from the theory of topological methods to moduli theory, cf. [3]; and, more specifically, the theory of Inoue-type varieties, introduced in [1]. In the theory of Inoue-type varieties, one can describe their moduli spaces explicitly in the case where the morphism $\phi_{0}$ has necessarily degree one onto its image. This is however a big restriction, and one would like to consider also the case where the morphism $\phi_{0}$ has degree at least two. We strive therefore towards a theory of multiple Inoue-type varieties and, in order to do this, we restrict ourselves in this paper to the special case where $\phi_{0}$ is a morphism which is generically finite onto its image, and where the canonical divisor of $W_{0}$ is ample.

To illustrate our main theorem, let us consider two simple examples, the first one where the image of $W_{0}$ is the smooth hypersurface $X:=\{\sigma=0\} \subset$ $\mathbb{P}^{n+1}, \sigma$ being a homogeneous polynomial of degree $d$. We let then $W_{0}$ be the complete intersection in the weighted projective space $\mathbb{P}(1,1, \ldots, 1, d)$ defined by the equations

$$
\begin{aligned}
& W_{0}=\left\{\left(z_{0}, z_{1}, \ldots, z_{n+1}, w\right) \mid \sigma\left(z_{0}, z_{1}, \ldots, z_{n+1}\right)=0\right. \\
& \left.\quad P\left(z_{0}, z_{1}, \ldots, z_{n+1}, w\right):=w^{m}+\sum_{i=1}^{m} w^{m-i} a_{i}\left(z_{0}, z_{1}, \ldots, z_{n+1}\right)=0\right\} .
\end{aligned}
$$

We can easily deform the complete intersection by deforming the degree $d$ equation adding a constant times the variable $w$, hence obtaining the following complete intersection:

$$
P\left(z_{0}, z_{1}, \ldots, z_{n+1}, w\right)=0, t w-\sigma\left(z_{0}, z_{1}, \ldots, z_{n+1}\right)=0, t \in \mathbb{C} .
$$

Clearly, for $t=0$ we obtain the previous $W_{0}$, a degree $m$ covering of the hypersurface $X=\{\sigma=0\}$, whereas for $t \neq 0$ we can eliminate the variable $w$ and obtain a hypersurface $W_{t}$ in $\mathbb{P}^{n+1}$ with equation (of degree $m d$ )

$$
P\left(z_{0}, z_{1}, \ldots, z_{n+1}, \sigma(z) / t\right)=0 \text {. }
$$

\section{Example 1.1. (Iterated weighted deformations).}

Now, one can iterate this process, and consider, in the weighted projective space

$$
\mathbb{P}\left(1,1, \ldots, 1, d, d m_{1}, \ldots, d m_{k}\right), \quad m_{1}\left|m_{2}\right| \ldots \mid m_{k},
$$

a complete intersection $W$ of multidegrees $\left(d, d m_{1}, \ldots, d m_{k}, d m\right)$, where $m_{k} \mid m=: m_{k+1}$.

Then, necessarily, there exist constants $t_{0}, t_{1}, \ldots, t_{k}$ such that the equations of $W$ have the following form, where the $Q_{j}$ 's are general weighted homogeneous polynomials of degree $=d m_{j}$ (in particular we assume them to be monic, so that the rational map to projective space is a morphism): 


$$
\left\{\begin{array}{l}
\sigma(z)=w_{0} t_{0} \\
Q_{1}\left(w_{0}, z\right)=w_{1} t_{1} \\
\ldots \quad \ldots \\
Q_{k}\left(w_{0}, \ldots, w_{k-1}, z\right)=w_{k} t_{k} \\
Q_{k+1}\left(w_{0}, \ldots, w_{k}, z\right)=0 .
\end{array}\right.
$$

Again, if all the $t_{j}$ 's are $\neq 0$, we can eliminate the variables $w_{j}$, and we obtain a hypersurface $\{F(z)=0\}$ in $\mathbb{P}^{n+1}$.

We claim that the above description generalizes, and the main idea of the following main theorem is that one can replace weighted projective space $\mathbb{P}\left(1,1, \ldots, 1, d, d m_{1}, \ldots, d m_{k}\right), m_{1}\left|m_{2}\right| \ldots \mid m_{k}$, by the total space of a direct sum of line bundles over some projective variety $X$, or $Z_{0} \supset X$, or over a family $\mathcal{Z}$ of projective varieties.

The first assertion of the main theorem is that, in order that $\phi_{0}: W_{0} \rightarrow Z_{0}$ deforms to a hypersurface embedding, a necessary condition is that $\phi_{0}$ : $W_{0} \rightarrow X:=\phi_{0}\left(W_{0}\right)$ is a smooth iterated univariate covering of normal type (see the next section for this very restrictive condition).

The main theorem also gives sufficient conditions, given such a covering, for the existence of a deformation to a hypersurface embedding.

We then give the proof, and in the final section, we discuss the first applications to the case where the target manifold $Z_{0}$ is projective space or an Abelian variety.

We defer the applications to the theory of multiple Inoue type varieties to a future paper.

We work of course over the complex numbers, and in several situations we consider also more general compact complex manifolds than projective manifolds.

\section{Statement of the main theorem}

To give a clear statement of our results, we need to introduce the following terminology.

\section{Definition 2.1.}

i) Given a complex space (or a scheme) $X$, a univariate covering of $X$ is a hypersurface $Y$, contained in a line bundle over $X$, and defined there as the zero set of a monic polynomial.

This means, $Y=\operatorname{Spec}(\mathcal{R})$, where $\mathcal{R}$ is the quotient algebra of the symmetric algebra over an invertible sheaf $\mathcal{L}, \operatorname{Sym}(\mathcal{L})=\oplus_{i \geq 0} \mathcal{L}^{\otimes i}$, by a monic (univariate) polynomial:

$$
\mathcal{R}:=\operatorname{Sym}(\mathcal{L}) /(P), P=w^{m}+a_{1}(x) w^{m-1}+a_{2}(x) w^{m-2}+\cdots+a_{m}(x) .
$$

Here $a_{j} \in H^{0}\left(X, \mathcal{L}^{\otimes j}\right)$. The univariate covering is said to be smooth if both $X$ and $Y$ are smooth.

ii) An iterated univariate covering $W \rightarrow X$ is a composition of univariate coverings

$$
f_{k+1}: W \rightarrow X_{k}, f_{k}: X_{k} \rightarrow X_{k-1}, \ldots, f_{1}: X_{1} \rightarrow X
$$


whose associated line bundles are denoted $\mathcal{L}_{k}, \mathcal{L}_{k-1}, \ldots, \mathcal{L}_{1}, \mathcal{L}_{0}$.

iii) In the case where $X \subset Z$ is a (smooth) hypersurface, we say that the iterated univariate covering is of normal type if

- all the line bundles $\mathcal{L}_{j}$ are pull back from $X$ of a line bundle of the form $\mathcal{O}_{X}\left(m_{j} X\right)$, and moreover

- $m_{1}\left|m_{2}\right| \ldots \mid m_{k}$, and the degree of $f_{j}$ equals $\frac{m_{j}}{m_{j-1}}$.

- we say that the iterated covering is normally induced if moreover all the coefficients $a_{I}(x)$ of the polynomials

$$
Q_{j}\left(w_{0}, \ldots, w_{j-1}, x\right)=\sum_{I} a_{I}(x) w^{I}
$$

describing the intermediate extensions are sections of a line bundle $\mathcal{O}_{X}(r(I) X)$ coming from $H^{0}\left(Z, \mathcal{O}_{Z}(r(I) X)\right)$.

Remark 2.2. The property that the iterated univariate covering $W \rightarrow X$ is normally induced clearly means that it is the restriction to $X$ of an iterated univariate covering of $Z$.

The property that the former is smooth does not necessarily imply that also the latter is smooth.

Definition 2.3. A 1-parameter deformation to hypersurface embedding consists of the following data:

(1) a one dimensional family of smooth projective varieties of dimension $n$ (i.e., a smooth projective holomorphic map $p: \mathcal{W} \rightarrow T$ where $T$ is a germ of a smooth holomorphic curve at a point $0 \in T$ ) mapping to another family $\pi: \mathcal{Z} \rightarrow T$ of smooth projective varieties of dimension $n+1$ via a relative map $\Phi: \mathcal{W} \rightarrow \mathcal{Z}$ such that $\pi \circ \Phi=p$ (hence we have the following commutative diagram)

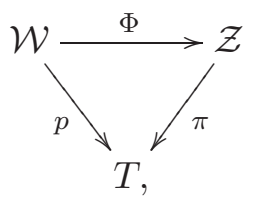

such that moreover

(2) for $t \neq 0$ in $T, \Phi_{t}$ is an embedding of $W_{t}:=p^{-1}(t)$ in $Z_{t}$,

(3) the restriction of the map $\Phi$ on $W_{0}$ is a generically finite morphism of degree $m$, so that the image of $\left.\Phi\right|_{W_{0}}$ is the cycle $\Sigma_{0}:=m X$ where $X$ is a reduced hypersurface in $Z_{0}$, defined by an equation $X=\{\sigma=0\}$.

Put in concrete terms, one can take a local coordinate $t$ for $T$ at 0 , and write, locally around $\{t=0\}$ the equation of the image $\Sigma:=\Phi(\mathcal{W})$ in $\mathcal{Z}$ via the Taylor series development in $t$, in terms of local coordinates $z=$ $\left(z_{1}, \ldots, z_{n}, z_{n+1}\right)$ on $Z_{0}$,

$$
\Sigma(z, t): \sigma(z)^{m}+t \sigma_{1}(z)+t^{2} \sigma_{2}(z)+\cdots+t^{m-1} \sigma_{m-1}(z)+\ldots=0 .
$$

$\mathcal{W}$ is a resolution of $\Sigma$ and the next theorems indicates exactly the sequence of blow-ups needed in order to obtain the resolution $\mathcal{W}$. 
Theorem 2.4. (A) Suppose we have a 1-parameter deformation to hypersurface embedding

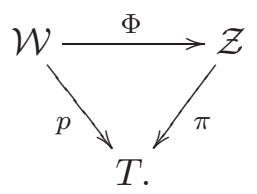

and assume that $K_{W_{0}}$ is ample.

Then we have:

(A1) $X$ is smooth,

(A2) There are line bundles $\mathcal{L}_{0}, \ldots, \mathcal{L}_{k}$ on $\mathcal{Z}$, such that $\left.\mathcal{L}_{j}\right|_{Z_{0}}=\mathcal{O}_{Z_{0}}\left(m_{j} X\right)$ for $j=0, \ldots, k$, with $1=m_{0}\left|m_{1}\right| m_{2} \ldots\left|m_{k}\right| m_{k+1}:=m$ (here $m$ is the degree of the morphism $\left.\Phi_{0}: W_{0} \rightarrow X\right)$, and such that $W_{0}$ is a complete intersection in $\left.\mathcal{L}_{0} \oplus \cdots \oplus \mathcal{L}_{k}\right|_{Z_{0}}$, with $\Phi_{0}$ a normally induced iterated smooth univariate covering .

(A3) $\mathcal{W}$ is obtained from $\Sigma:=\Phi(\mathcal{W})$ by a finite sequence of blow-ups.

Moreover the local equations of $\mathcal{W}$ are of the following standard form

$$
\left\{\begin{array}{l}
\sigma(z)=w_{0} t \\
Q_{1}\left(w_{0}, z\right)=w_{1} t \\
\ldots \quad \ldots \\
Q_{k}\left(w_{0}, \ldots, w_{k-1}, z\right)=w_{k} t \\
Q_{k+1}\left(w_{0}, \ldots, w_{k}, z, t\right)=0 .
\end{array}\right.
$$

(B1) Conversely, take any smooth iterated univariate covering of normal type

$$
\phi_{0}: W_{0} \rightarrow X \subset Z_{0}
$$

and take any 1-parameter family $\mathcal{Z}$ of deformations of $Z_{0}$.

Then the line bundle $\mathcal{O}_{Z_{0}}(X)$ extends to a line bundle $\mathcal{L}_{0}$ on the whole family $\mathcal{Z}$. And $W_{0}$ deforms to a hypersurface embedding if, for all $i \geq$ 2 , every section in $H^{0}\left(Z_{0}, \mathcal{O}_{Z_{0}}(i X)\right)$ and every section in $H^{0}\left(X, \mathcal{O}_{X}(i X)\right)$ comes from a section in $H^{0}\left(\mathcal{Z}, \mathcal{L}_{0}^{\otimes i}\right)$.

(B2) This holds in particular, when the family $\mathcal{Z}$ is trivial, $\mathcal{Z} \cong Z_{0} \times T$, if the necessary condition of being normally induced is fulfilled.

Remark 2.5. (b1) More precisely, in (B1) above, there is a family $\mathcal{W}$ such that $\mathcal{W}$ is a complete intersection in $\mathcal{L}_{0} \oplus \cdots \oplus \mathcal{L}_{k}\left(\mathcal{L}_{i}=\mathcal{L}_{0}^{\otimes m_{i}}\right)$, and $\mathcal{W}$ is given as above; moreover, for $t \neq 0$ in $T$, the morphism $\Phi_{t}$, induced on $W_{t}$ by the bundle projection on $Z_{t}$, is an embedding.

(b2) sufficient condition in (B2) is the surjectivity of $H^{0}\left(Z_{0}, \mathcal{O}_{Z_{0}}(i X)\right) \rightarrow$ $H^{0}\left(X, \mathcal{O}_{X}(i X)\right)$ for $i \geq 2$; this is implied by $H^{1}\left(Z_{0}, \mathcal{O}_{Z_{0}}(i X)\right)=0, \forall i \geq 1$.

Remark 2.6. The line bundle $\mathcal{O}_{Z_{0}}(X)$ extends to a line bundle $\mathcal{L}_{0}$ on the whole family $\mathcal{Z}$, because of the Lefschetz $(1,1)$ theorem, since $\mathcal{O}_{Z_{0}}(m X)$ does.

Observe moreover that there is a (non-canonical) isomorphism

$$
\operatorname{Pic}^{0}\left(Z_{0}\right) \cong \operatorname{Pic}^{0}\left(Z_{t}\right)
$$

whereas in general there is no isomorphism of $\operatorname{Pic}\left(Z_{0}\right)$ with $\operatorname{Pic}\left(Z_{t}\right)$. 
Remark 2.7. (i) Consider the family of submanifolds of weighted projective spaces given in example 1.1, with equations I-2, and consider the 1-parameter deformation where we set $t_{j}=t^{n_{j}}$.

Then the equations considered in the proof of the theorem are many more than equations [-2, since for instance from the equation $\sigma(z)=t^{n_{0}} w_{0}$ we recover $w_{0}$ not directly but only after an iterated procedure: we inductively set $\sigma(z)=t^{a} v_{a-1}$, so finally we get $w_{0}=v_{n_{0}-1}$.

\section{Auxiliary Results And proof of the main theorem}

The following observation plays an important role in the proof.

Lemma 3.1. Suppose we have a one dimensional smooth family $p: \mathcal{W} \rightarrow T$ of smooth projective varieties of dimension $n$ mapping to another flat family $q: \mathcal{Y} \rightarrow T$ of projective varieties of the same dimension via a relative map $\Psi: \mathcal{W} \rightarrow \mathcal{Y}$ over a smooth holomorphic curve $T$ such that $q \circ \Psi=p$, i.e. we have the commutative diagram

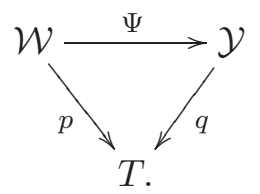

Assume that

(1) $\mathcal{Y}$ is normal and Gorenstein,

(2) $\Psi$ is birational,

(3) for $t \neq 0$ in $T, \Psi$ induces an isomorphism,

(4) $K_{W_{0}}$ is ample.

Then we have that $\Psi$ is an isomorphism, in particular $W_{0} \cong Y_{0}$.

Proof. We have $K_{\mathcal{W}}=\Psi^{*}\left(K_{\mathcal{Y}}\right)+\mathcal{B}$. Since we assume that $\Psi$ induces an isomorphism for $t \neq 0$ in $T$, the support of the Cartier divisor $\mathcal{B}$ is contained in $W_{0}$, which is irreducible.

Now $Y_{0}$ has dimension $n$ and the morphism $\Psi_{0}: W_{0} \rightarrow Y_{0}$ is generically finite, hence we conclude that $\mathcal{B}=0$. In particular, $K_{\mathcal{W}}=\Psi^{*}\left(K_{\mathcal{Y}}\right)$; restricting to the special fibre, we obtain $K_{W_{0}}=\Psi_{0}^{*}\left(K_{Y_{0}}\right)$. Since by assumption $K_{W_{0}}$ is ample, $\Psi_{0}$ is finite, hence also $\Psi$ is finite, hence an isomorphism in view of the normality of $\mathcal{Y}$.

Remark 3.2. Without the assumption that $K_{W_{0}}$ is ample one can only assert that $Y_{0}$ is normal with at most canonical singularities.

Lemma 3.3. In the hypotheses of theorem 2.4, we have that $\sigma^{m-i} \mid \sigma_{i}$ for $i=1, \ldots, m-1$.

Proof. Since the map $W_{0} \rightarrow X$ is a generically finite map of degree $m$, given a general point $p$ of $X$, the inverse image of $p$ consists of $m$ points $q_{1}, \ldots, q_{m}$, and at each $q_{i}$ the rank of the derivative of the morphism $W_{0} \rightarrow Z_{0}$ is equal to $n$. Hence we get local coordinates $\left(w_{1}, \ldots, w_{n}, t\right)$ for $\mathcal{W}$ at $q_{i}$ and local coordinates $\left(z_{1}, \ldots, z_{n}, z_{n+1}, t\right)$ for $\mathcal{Z}$ at $p$ (not depending on $i$ ) such that $\Phi$ is given by a function $f_{i}$

$$
f_{i}\left(w_{1}, \ldots, w_{n}, t\right)=\left(w_{1}, \ldots, w_{n}, \varphi_{i}\left(w_{1}, \ldots, w_{n}, t\right), t\right)
$$


such that

$$
f_{i}\left(w_{1}, \ldots, w_{n}, 0\right)=\left(w_{1}, \ldots, w_{n}, 0,0\right) .
$$

Here $\varphi_{i}\left(w_{1}, \ldots, w_{n}, t\right)$ is a holomorphic function.

Hence at $p \in X$, there are variables $\left(z_{1}, \ldots, z_{n}, z_{n+1}, t\right)$ such that $\sigma=$ $z_{n+1}$, and $\Sigma$ consists of $m$ smooth branches with equation

$$
\sigma-\varphi_{i}\left(z_{1}, \ldots, z_{n}, t\right)=0, \sigma=z_{n+1}
$$

for $i=1, \ldots, m$.

Hence, setting $z:=\left(z_{1}, \ldots, z_{n}\right)$ the local equation of $\Sigma$ is

$$
\prod_{i=1}^{m}\left(\sigma-\varphi_{i}(z, t)\right)=\sigma^{m}-a_{1}(z, t) \sigma^{m-1}+\ldots+a_{m}(z, t)
$$

and $t^{i} \mid a_{i}$, since $t \mid \varphi_{i}$.

Remark 3.4. Since we assume that $\Phi_{t}$ is an embedding when $t \neq 0$, for a fixed value of $z:=\left(z_{1}, \ldots, z_{n}\right)$ equation III-1 yields a plane curve with $m$ smooth branches. Equivalently, we may view equation III-1 as giving a plane curve over a non algebraically closed field (the fraction field of the ring of power series in $z$ ).

Lemma 3.5. Assume the same hypotheses of part (A) of theorem 2.4 with $n=1$. Let $p \in W_{0}$ be any point. Then there is a neighborhood $U$ of $p$ in $W_{0}$ such that $\phi_{0}(U)$ is a smooth curve of $Z_{0}$ at $\phi_{0}(p)$.

Proof. We have a factorization $\phi_{0}=\nu \circ \psi_{0}$ near the point $p$

$$
W_{0} \stackrel{\psi_{0}}{\longrightarrow} X^{\text {nor }} \stackrel{\nu}{\longrightarrow} X
$$

where $\nu: X^{\text {nor }} \rightarrow X$ is the normalization.

There are respective local coordinates $w$ around $p \in W_{0}, u$ around $\psi_{0}(p)$, $x, y$ around $\phi_{0}(p)$ such that:

- $\psi_{0}(w)=w^{h}=u$,

- $x(u), y(u)$ are a Puiseux parametrization of the branch associated to $\phi_{0}(p)$. If the branch is nonsingular, without loss of generality $y \equiv 0$ and there is nothing to prove.

- Otherwise the branch is singular, and we have a Puiseux parametrization of the form

$$
\left\{\begin{array}{l}
x=u^{d} \\
y=u^{e}+g(u) u^{e+1}, \text { where } e>d .
\end{array}\right.
$$

Hence the branch is the zero set of a pseudo-polynomial in $x$,

$$
x^{d}+a_{1}(y) x^{d-1}+\cdots+a_{d}(y) .
$$

Now, we can write, locally identifying $\mathcal{Z}$ to $\mathbb{C}^{2} \times T, \Phi(w, t)$ as

$$
\Phi(w, t)=(\phi(w, t), t)
$$

and $\phi(w, t)$ as

$$
\left\{\begin{array}{l}
x=w^{d h}+t \phi_{1}(w, t) \\
y=w^{e h}\left(1+g\left(w^{h}\right) w^{h}\right)+t \phi_{2}(w, t)
\end{array}\right.
$$


The link of the branch $\phi_{0}(p)$ is, by Zariski's theorem [9], an iterated nontrivial toral knot, running $h$ times. However, $L_{0}$ is isotopic to $L_{t}$, which is gotten by the image of the circle $|w|=\epsilon$ under $\phi(w, t)$.

Observe that the map $\phi(w, t)$, by purity of branch locus, ramifies on a curve $R$, which is not contained in $\{t=0\}$ : since $W_{t}$ is embedded for $t \neq 0$, it follows that $R$ is exceptional and that the curves $W_{t}$ are a family of curves through the origin $x=y=0 \in \mathbb{C}^{2}$. Moreover, again since $W_{t}$ embeds, the reduced curve $R_{\text {red }}$ is a smooth curve which projects isomorphically to the $t$-axis, so we may assume without loss of generality that $R_{\text {red }}=\{w=0\}$.

The link $L_{t}$ is contained in the submanifold $W_{t}$ of a four dimensional ball $B$ around the origin; $W_{t}$ is a smooth holomorphic curve through the point $\phi(0, t)=0$, hence $L_{t}$ yields an unknotted circle $S^{1} \subset S^{3}$. We have derived a contradiction from assuming $d>1$, and that the branch is singular.

Lemma 3.6. Assume the same hypotheses of Theorem 2.4. Let

$$
W_{0}^{f}=\left\{p \in W_{0} \mid \phi_{0} \text { is finite at } p\right\} .
$$

Then if $p \neq p^{\prime} \in W_{0}^{f}$ and $\phi_{0}(p)=\phi_{0}\left(p^{\prime}\right)=y_{0}$, then the germs of analytic subsets $Y_{0}:=\phi_{0}\left(U_{p}\right)$ and $Y_{0}^{\prime}:=\phi_{0}\left(U_{p^{\prime}}\right)$ coincide.

Proof. Cut now $\mathbb{C}^{n+1}$ with a general linear $\mathbb{C}^{2}$ passing through $y_{0}$, so that we get curves $\mathbb{C}^{2} \cap Y_{0}=C, \mathbb{C}^{2} \cap Y_{0}^{\prime}=C^{\prime}$, passing through $y_{0}$. Now $\left(C \cdot C^{\prime}\right)_{y_{0}}=$ $d \geq 1$, and indeed $d$ is a topological invariant, it is the linking number of $C \cap S^{3}$ and $C^{\prime} \cap S^{3}$.

For $|t|<<1$, we can deform the curves $C, C^{\prime}$ which are in the image of $W_{0}$, to curves $C(t), C^{\prime}(t)$ in the image of $W_{t}$ : these have the property that in a neighborhood of $y_{0} \in \mathbb{C}^{2}$ they intersect in $d$ points, counted with multiplicities. Hence, if $y(t) \in C(t) \cap C^{\prime}(t)$, then there are $p(t) \neq p^{\prime}(t) \in W_{t}$ with $\phi_{0}(p(t))=\phi_{0}\left(p^{\prime}(t)\right)$. This is a contradiction.

Proposition 3.7. Assume the same hypotheses of Theorem 2.4. Then $X$ is normal.

Proof. $X$ is a hypersurface in a smooth manifold, hence it is normal if and only if it is smooth outside of codimension 2 in $X$.

The image of the points in $W_{0} \backslash W_{0}^{f}$ is a Zariski closed subset of codimension $\geq$ 2 in $X$.

Hence it suffices to consider points $q \in X$ which are image points only of points $p \in W_{0}^{f}$.

By Lemma 3.6 the germ of $X$ at $q$ equals the image of the germ of $W_{0}$ at $p$.

Let $\mathcal{C}_{k}=\left\{p \in W_{0} \mid \operatorname{rank}\left(D \phi_{0}\right)_{p}=k\right\}$. Then $\phi_{0}\left(\mathcal{C}_{k}\right)$ has dimension $\leq n-2$ for $k \leq n-2$, and we conclude that $X$ is smooth outside of codimension 2 unless $\operatorname{dim} \phi_{0}\left(\mathcal{C}_{n-1}\right)=n-1$.

It also suffices to consider the general point $p$ of $\mathcal{C}:=\mathcal{C}_{n-1}$, where $\mathcal{C}$ is smooth of dimension $n-1$ and $\operatorname{rank}\left(D\left(\phi_{0} \mid \mathcal{C}\right)\right)=n-1$.

Let $(\hat{d}-1)$ be the multiplicity of $\mathcal{C}$ in the locus given by the $n \times n$ minors of the derivative matrix. 
There are local coordinates $(v, w)$ in a neighborhood of $p \in W_{0}$, with $v=\left(v_{1}, \ldots, v_{n-1}\right)$, such that $\mathcal{C}=\{w=0\}$ and such that

$$
\phi_{0}(v, w)=(v, x(v, w), y(v, w)) .
$$

The locus given by the $n \times n$ minors of the derivative matrix is then just the locus $\frac{\partial x}{\partial w}=0, \frac{\partial y}{\partial w}=0$.

Without loss of generality, at the general point of $\mathcal{C}$ we can assume that

$$
x(v, w)=\sum_{i=\hat{d}}^{\infty} a_{i}(v) w^{i},
$$

and, since $a_{\hat{d}}(x) \not \equiv 0$, we may assume that $a_{\hat{d}}(0) \neq 0$. Hence we choose coordinates $x, y$ with

$$
\left\{\begin{array}{l}
x=w^{\hat{d}}, \\
y=w^{e}+\cdots \text { with } e>\hat{d} \text { and } \hat{d} \backslash e .
\end{array}\right.
$$

Then, if $y \not \equiv 0$, we get, for any $v$ in a neighborhood of 0 , a singular curve branch. The same argument as for the case $n=1$ applied to $x(0, w), y(0, w)$ gives a contradiction. So we have established the proof.

Lemma 3.8. Let $C$ be a germ of a plane curve singularity consisting of $m$ smooth branches with non vertical tangents, i.e. the local equation of $C$ is $F(y, t)=0$, with

$$
\begin{aligned}
& F(y, t):= \\
& =\prod_{i=1}^{m}\left(y-\varphi_{i}(t)\right) \\
& =y^{m}-\sigma_{1}\left(\varphi_{1}, \ldots, \varphi_{m}\right) y^{m-1}+\sigma_{2}\left(\varphi_{1}, \ldots, \varphi_{m}\right) y^{m-2}+\cdots+(-1)^{m} \varphi_{1} \cdots \varphi_{m} .
\end{aligned}
$$

Here, $\sigma_{i}$ is the $i$-th elementary symmetric function, and $t \mid \varphi_{i}(t)$, since $(0,0)$ is the singular point, hence $t^{i}$ divides $\sigma_{i}\left(\varphi_{1}, \ldots, \varphi_{m}\right)$.

Then the singularity can be resolved by iterated blow-ups of the form:

$$
\left\{\begin{array}{l}
y=w_{0} t \\
D_{1}\left(w_{0}\right)=w_{1} t \\
D_{2}\left(w_{1}\right)=w_{2} t \\
\cdots \quad \cdots \\
D_{k}\left(w_{k-1}\right)=w_{k} t
\end{array}\right.
$$

where the $D_{j}$ 's are monic polynomials.

Proof. $F(y, t)$ is a pseudo-polynomial. Write

$$
F(y, t)=y^{m}+t b_{1}(t) y^{m-1}+\cdots+t^{m} b_{m}(t) .
$$

The first blow-up yields

$$
P\left(w_{0}, t\right)=w_{0}^{m}+b_{1}(t) w_{0}^{m-1}+\cdots+b_{m}(t) .
$$

Let $P\left(w_{0}, t\right)=P_{0}\left(w_{0}\right)+t P_{1}\left(w_{0}\right)+t^{2} P_{2}\left(w_{0}\right)+\cdots$ where $P_{0}\left(w_{0}\right)$ is a monic polynomial of degree $m$ and $\operatorname{deg}_{w_{0}} P_{j}\left(w_{0}\right) \leq m-1$ for $j \geq 1$. Looking at the two partial derivatives for $t=0$, the proper transform $C_{0}$ is smooth if and only if

$$
P_{0}\left(w_{0}\right)=0, \frac{\partial}{\partial w_{0}} P_{0}\left(w_{0}\right)=0, P_{1}\left(w_{0}\right)=0
$$


have no common roots.

If not, let $D_{1}\left(w_{0}\right)=$ gcd of the above three polynomials, so that $P_{0}\left(w_{0}\right)=$ $D_{1}^{r} \cdot G$ for some $2 \leq r \leq m$ such that $r \cdot \operatorname{deg}\left(D_{1}\right) \leq m$.

To continue, observe that $D_{1}$ is again monic, so there is no tangent $t=0$, and it suffices to blow-up $t=D_{1}=0$ setting $D_{1}\left(w_{0}\right)=w_{1} t$.

We get equations

$$
\begin{aligned}
& D_{1}\left(w_{0}\right)^{r} \cdot G\left(w_{0}\right)+t D_{1}\left(w_{0}\right) \tilde{P}\left(w_{0}\right)+t^{2} \cdots \\
& =t^{r}\left[w_{1}^{r} \cdot G\left(w_{0}\right)+w_{1}^{r-1} \cdots\right] .
\end{aligned}
$$

where the divisibility of the second term by $w_{1}^{r-1}$, and similarly for the next terms follows by applying once more Lemma 3.3 .

We continue this process until all branches are separated.

\section{Proof of Theorem 2.4}

Recall the Taylor series development in $t$ of the equation in $\mathcal{Z}$ of the image $\Sigma:=\Phi(\mathcal{W}):$

$$
\Sigma(z, t):=\sigma(z)^{m}+t \sigma_{1}(z)+t^{2} \sigma_{2}(z)+\cdots+t^{m-1} \sigma_{m-1}(z)+\ldots
$$

Choose a general point $p$ in $X$. In order to show that the process terminates we shall at a later moment consider a germ of plane curve $C$ passing through $p$, consisting of $m$ smooth branches with non vertical tangents, and obtained as a linear section of $\Sigma$ (i.e., $C$ is obtained by setting $\left(z_{1}, \ldots, z_{n}\right)$ $=$ constant in appropriate local coordinates).

Observe that, by Lemma 3.3. $\sigma^{i} \mid \sigma_{m-i}$ for $i=1, \ldots, m-1$. So we can rewrite the equation of $\Sigma$ as follows:

$$
\Sigma(z, t):=\sigma(z)^{m}+a_{1}(z, t) \sigma(z)^{m-1}+a_{2}(z, t) \sigma(z)^{m-2}+\cdots+a_{m}(z, t)=0
$$

where $t^{i} \mid a_{i}(z, t)$.

Please observe that the above equation is not just a local equation, but that it is a global equation for a section of a line bundle on $\mathcal{Z}$ (see remark 2.6)

$$
\mathcal{O}_{\mathcal{Z}}(\Sigma)=\mathcal{L}_{0}^{\otimes m}
$$

Now, by setting $\sigma=t w_{0}$ and $a_{i}(z, t)=t^{i} b_{i}(z, t)$ in equation III-2, we obtain the equation

$$
P_{w_{0}}\left(w_{0}, z, t\right):=w_{0}^{m}+b_{1}(z, t) w_{0}^{m-1}+\cdots+b_{m}(z, t)=0 .
$$

This is a hypersurface and its singular locus is contained in $t=0$ by our assumption. Write

$$
b_{j}(z, t)=b_{j, 0}(z)+t b_{j, 1}(z)+\cdots,
$$

so that the $b_{j, 0}(z)$ 's are sections on $Z_{0}$ of a line bundle of the form $\mathcal{O}_{Z_{0}}(i X)$ (this observation shall be repeated in the sequel, leading to the proof that we get a normally induced covering of $X$ ).

Hence we can write

$$
P\left(w_{0}, z\right):=w_{0}^{m}+b_{1,0}(z) w_{0}^{m-1}+\cdots+b_{m, 0}(z) .
$$


Hence the equation $P_{w_{0}}$ has the development

$$
P_{w_{0}}\left(w_{0}, z, t\right)=P\left(w_{0}, z\right)+t \sum_{j=1}^{m} b_{j, 1} w_{0}^{m-j}+t^{2} \cdots .
$$

Set $\hat{P}\left(w_{0}, z\right):=\sum_{j=1}^{m} b_{j, 1} w_{0}^{m-j}$; then the gradient of $P_{w_{0}}$ for $t=0$ equals

$$
\left(\frac{\partial P}{\partial w_{0}}, \hat{P}, \frac{\partial P}{\partial z_{1}}, \frac{\partial P}{\partial z_{2}}, \ldots\right) .
$$

The singular locus is contained in $t=0$ and is there given by

$$
\left\{\begin{array}{l}
P\left(w_{0}, z\right)=0 \\
\frac{\partial}{\partial w_{0}} P\left(w_{0}, z\right)=0 \\
\hat{P}\left(w_{0}, z\right)=0 \\
\frac{\partial}{\partial z_{i}} P\left(w_{0}, z\right)=0(\text { for } i=1, \ldots, n) .
\end{array}\right.
$$

The hypersurface defined by $P_{w_{0}}\left(w_{0}, z, t\right)=0$ is normal unless it is singular in codimension 1. Assume that the hypersurface defined by $P_{w_{0}}\left(w_{0}, z, t\right)=$ 0 is not normal, so that it is singular in codimension 1 . Observe that the square free part of $P\left(w_{0}, z\right)$ is irreducible because its zero set is the image of an irreducible variety $W_{0}$, hence for $t=0$ the vanishing of $P\left(w_{0}, z\right)$ should imply the vanishing of the other polynomials.

Since $P\left(w_{0}, z\right)=0 \Rightarrow \partial P\left(w_{0}, z\right) / \partial w_{0}=0$, hence $P\left(w_{0}, z\right)=Q\left(w_{0}, z\right)^{r}$, for some $2 \leq r \leq m$.

And $Q\left(w_{0}, z\right)$ is again irreducible and $Q\left(w_{0}, z\right) \mid \hat{P}\left(w_{0}, z\right)$. Now, since $r \geq$ 2 ,

$$
\partial P\left(w_{0}, z\right) / \partial z_{i}=r Q\left(w_{0}, z\right)^{r-1} \partial Q\left(w_{0}, z\right) / \partial z_{i},
$$

so the last conditions are automatically fulfilled.

If we write the equation $P_{w_{0}}\left(w_{0}, z, t\right)$ in terms of $Q\left(w_{0}, z\right)$, then

$$
Q\left(w_{0}, z\right)^{r}+t Q\left(w_{0}, z\right) \tilde{P}\left(w_{0}, z, t\right)+t^{2} \cdots .
$$

Then again by Lemma 3.3, $Q\left(w_{0}, z\right)^{r-2} \mid \tilde{P}(w, z, t)$, and so on.

Observe that $Q\left(w_{0}, z\right)=0$ gives a covering $W_{0}^{\prime}$ of $X$ of degree $m / r$.

In view of Lemma 3.8, we set $Q\left(w_{0}, z\right)=w_{1} t$. Then we get an iterated covering as in Lemma 3.8, where $P=D_{1}^{r}=Q^{r}$. We consider now a germ of plane curve $C$ through the general point $p \in X$ consisting of $m$ smooth branches with non vertical tangents by taking linear sections. Since the resolution of $C$ is obtained by a finite sequence of blow-ups, we get an iterated covering as in Lemma 3.8, which is normal and a complete intersection.

Lemma 3.1 implies that we have then obtained $\mathcal{W}$.

Since $W_{0}$ is smooth, let us set $t=0$ in the above equations [I-1, and observe that the matrix of derivatives has triangular form. So, smoothness of $W_{0}$ implies the smoothness of $X$ and of all the intermediate coverings.

Therefore we have shown (A1), (A2) and (A3).

Let us show now the converse, (B1) and (B2).

Assume we are given an iterated smooth univariate covering $W_{0}$ of $X$ which is normally induced, defined by equations: 
(III-3)

$$
\left\{\begin{array}{l}
\sigma(z)=0 \\
Q_{1}\left(w_{0}, z\right)=0 \\
\ldots \quad \ldots \\
Q_{k}\left(w_{0}, \ldots, w_{k-1}, z\right)=0 \\
Q_{k+1}\left(w_{0}, \ldots, w_{k}, z\right)=0 .
\end{array}\right.
$$

Claim I): if $H^{0}\left(Z_{0}, \mathcal{O}_{Z_{0}}(i X)\right)$ surjects onto $H^{0}\left(X, \mathcal{O}_{X}(i X)\right)$ for each $i \geq 2$, then every iterated univariate covering $W_{0}$ of $X$ is normally induced, i.e., it extends to an iterated univariate covering of $Z_{0}$.

Proof. First of all we can put the equations of the iterated covering $W_{0}$ of $X$ in Tschirnhausen form. Here the polynomial equation of a univariate covering is said to be in Tschirnhausen form if $a_{1}(x) \equiv 0$, and every covering can be put in Tschirnhausen form after an automorphism replacing $w$ with $w-\frac{1}{m} a_{1}(x)$.

Second, the coefficients $a_{j, I}$ of the polynomials $Q_{j}$ are now given by sections of line bundles of the form $\mathcal{O}_{X}(n(I, j) X)$, where $n(I, j) \geq 2$. By assumption, they extends to sections of $\mathcal{O}_{Z_{0}}(n(I, j) X)$.

That this holds if $H^{1}\left(Z_{0}, \mathcal{O}_{Z_{0}}(i X)\right)=0, \forall i \geq 1$, follows immediately from the long exact cohomology sequence associated to the exact sequence

$$
0 \rightarrow \mathcal{O}_{Z_{0}}(i X) \rightarrow \mathcal{O}_{Z_{0}}((i+1) X) \rightarrow \mathcal{O}_{X}((i+1) X) \rightarrow 0,
$$

for $i \geq 1$.

Let's pass to the proof of (B1).

By our assumption, $\sigma(z)$ extends to a section $\sigma(z, t)$ of the line bundle $\mathcal{L}_{0}$ on the whole family $\mathcal{Z}$. Similarly the sections $a_{j, I}$ extend to sections $a_{j, I}(z, t)$ over $\mathcal{Z}$, hence we can also extend the polynomials $Q_{j}\left(w_{0}, \ldots, w_{j-1}, z\right)$ to polynomials $Q_{j}\left(w_{0}, \ldots, w_{j-1}, z, t\right)$.

Then we define the iterated univariate covering $\mathcal{W}$ of $\mathcal{Z}$ via the following equations:

$$
\left\{\begin{array}{l}
\sigma(z, t)=w_{0} t \\
Q_{1}\left(w_{0}, z, t\right)=w_{1} t \\
\cdots \quad \ldots \\
Q_{k}\left(w_{0}, \ldots, w_{k-1}, z, t\right)=w_{k} t \\
Q_{k+1}\left(w_{0}, \ldots, w_{k}, z, t\right)=0 .
\end{array}\right.
$$

To finish the proof of (B2), observe that if the family $\mathcal{Z}$ is trivial, $\mathcal{Z}=$ $Z_{0} \times T$, then obviously

$$
H^{0}\left(Z_{0}, \mathcal{O}_{Z_{0}}(i X)\right) \subset H^{0}\left(\mathcal{Z}, \mathcal{O}_{\mathcal{Z}}(i X)\right),
$$

hence there is no problem to extend the iterated univariate covering to one of $\mathcal{Z}$. 


\section{Applications}

The first applications that we shall give are, more or less, direct corollaries of the previous general results.

\subsection{Hypersurfaces in projective space.}

Corollary 4.1. The smooth manifolds $W_{0}$ with ample canonical divisor which admit a 1-parameter deformation to a hypersurface embedding

$$
p: \mathcal{W} \rightarrow T, \quad \Phi: \mathcal{W} \rightarrow \mathbb{P}^{n+1} \times T
$$

(here, for $t \neq 0, W_{t}$ is a smooth hypersurface in $\mathbb{P}^{n+1}$ ) are exactly the iterated weighted deformations of example 1.1] with $m d>n+2$, and the family $\mathcal{W}$ is a pull back from the family in I-2.

The class of such manifolds $W_{0}$ is open in the Kuranishi space for $n \geq 2$.

Proof. Either $W_{0}$ is a hypersurface, and there is nothing to prove (set $m=1$ ), or the degree of $\phi_{0}: W_{0} \rightarrow X$ is $m \geq 2$.

Then theorem 2.4 applies, and it is easy to see that we get a manifold in the family [-2. The converse is direct (set $t=t_{j} \forall j$ in the family I-2).

The proof of the second statement follows imitating quite closely Sernesi's argument in [7] for the case of complete intersections in weighted projective spaces. 1

\subsection{Hypersurfaces in an Abelian variety.}

Corollary 4.2. The smooth manifolds with ample canonical divisor $W_{0}$ which admit a 1-parameter deformation $p: \mathcal{W} \rightarrow T$ where, for $t \neq 0, W_{t}$ is a smooth hypersurface in an Abelian variety $A_{t}$, are, for $n \geq 2$, exactly the iterated smooth univariate coverings $W_{0} \rightarrow X$ of normal type, where $X$ is an ample divisor in an Abelian variety $A_{0}$.

Proof. $A_{t}$ is, for $t \neq 0$, the Albanese variety of $W_{t}$, since $H^{i}\left(\mathcal{O}_{A_{t}}\left(-W_{t}\right)\right)=$ 0 for $i=1,2$, hence the exact cohomology sequence associated to the exact sequence

$$
0 \rightarrow \mathcal{O}_{A_{t}}\left(-W_{t}\right) \rightarrow \mathcal{O}_{A_{t}} \rightarrow \mathcal{O}_{W_{t}} \rightarrow 0
$$

yields $h^{1}\left(\mathcal{O}_{A_{t}}\right)=h^{1}\left(\mathcal{O}_{W_{t}}\right)$.

We obtain therefore a morphism

$$
\Phi: \mathcal{W} \rightarrow \mathcal{A}, A_{t}=\operatorname{Alb}\left(W_{t}\right)
$$

that induces a 1-parameter deformation to hypersurface embedding in Abelian varieties by lemma 149 of 3 .

Our main theorem applies, in particular (B2) holds since we have $H^{1}\left(\mathcal{O}_{A_{0}}(i X)\right)=0$ for all $i \geq 1$.

\footnotetext{
${ }^{1}$ In the more general situation of theorem 2.4 one can use Horikawa's theory of deformations of finite holomorphic maps, see [4, also the lecture notes [2].
} 
4.3. Multiple Inoue-type varieties. We can now extend the definition of Inoue type variety (see [1] and [3]) in the following way:

Definition 4.3. A primary multiple Inoue-type variety $W_{0}$ of smoothing type is a normally induced smooth iterated univariate covering $W_{0} \rightarrow X$, where $X$ is a smooth ample subvariety of a projective classifying space $Z_{0}$.

A multiple Inoue-type variety $Y_{0}$ of smoothing type is a quotient $W_{0} / G$, for the free action of a finite group, of a primary multiple Inoue-type variety $W_{0}$ of smoothing type.

We do not give further applications here, hopefully in a future paper.

\section{REFERENCES}

[1] [BC12] I. Bauer and F. Catanese, Inoue type manifolds and Inoue surfaces: a connected component of the moduli space of surfaces with $K^{2}=7, p_{g}=0$, Geometry and arithmetic, 23-56, EMS Ser. Congr. Rep., Eur. Math. Soc., Zürich, (2012).

[2] [Cat88] F. Catanese, Moduli of algebraic surfaces, 'Theory of moduli', Lecture Notes in Math., 1337, Springer, Berlin, 1- 83 (1988).

[3] [Cat15] F. Catanese, Topological methods in moduli theory. Bull. Math. Sci. 5, no. $3,287-449 .(2015)$.

[4] [Hor73] E. Horikawa, On deformations of holomorphic maps. I. J. Math. Soc. Japan 25 (1973), 372-396.

[5] [Hor75] E. Horikawa, On deformations of quintic surfaces. Invent. Math. 31, 43-85, (1975).

[6] [KS58] K. Kodaira and D. C. Spencer On deformations of complex analytic structures. II. Ann. of Math. (2) 67, 403-466, (1958).

[7] [Ser75] E. Sernesi, Small deformations of global complete intersections. Boll. Un. Mat. Ital. (4) 12 , no. 1-2, 138-146, (1975).

[8] [Ser15] E. Sernesi, General curves on algebraic surfaces. J. Reine Angew. Math. 700, 209-233, (2015).

[9] [Zar32] O. Zariski, On the topology of algebroid singularities. Amer. J. of Math. 54, 453-465 (1932).

Lehrstuhl Mathematik VIII, Mathematisches Institut der Universität Bayreuth, NW II, Universitätsstr. 30, 95447 Bayreuth, Germany

E-mail address: fabrizio.catanese@uni-bayreuth.de

Department of Mathematical Sciences, KAist, 291, Daehak-ro, YuseongGu, Daejeon, 34141, Korea

E-mail address: ynlee@kaist.ac.kr 